\title{
Wind Power Forecasting using an Artificial Neural Network for ASPCS
}

\author{
Kazuma Hanada ${ }^{1}$, Takataro Hamajima ${ }^{1}$, Makoto Tsuda $^{2}$, Daisuke Miyagi ${ }^{2}$, Takakazu Shintomi ${ }^{3}$, \\ Tomoaki Takao ${ }^{4}$, Yasuhiro Makida ${ }^{5}$, Masataka Kajiwara ${ }^{6}$ \\ ${ }^{1}$ Department of Electrical and Electronics Systems, Hachinohe Institute of Technology, Aomori, Japan \\ ${ }^{2}$ Department of Electrical Engineering, Tohoku University, Miyagi, Japan \\ ${ }^{3}$ Advanced Research Institute for the Sciences and Humanities, Nihon University, Tokyo, Japan \\ ${ }^{4}$ Department of Engineering and Applied Sciences, Sophia University, Tokyo, Japan \\ ${ }^{5}$ High Energy Accelerator Research Organization, Ibaraki, Japan \\ ${ }^{6}$ Iwatani Corporation, Tokyo, Japan \\ Email: hanada@hi-tech.ac.jp
}

Received April, 2013

\begin{abstract}
In order to use effectively renewable energy sources, we propose a new system, called Advanced Superconducting Power Conditioning System (ASPCS) that is composed of Superconducting Magnetic Energy Storage (SMES), Fuel Cell-Electrolyzer (FC-EL), hydrogen storage and DC/DC and DC/AC converters in connection with a liquid hydrogen station for fuel cell vehicles. The ASPCS compensates the fluctuating electric power of renewable energy sources such as wind and photovoltaic power generations by means of the SMES having characteristics of quick response and large Input-Output power, and hydrogen energy with FC-EL having characteristics of moderate response and large storage capacity. The moderate fluctuated power of the renewable energy is compensated by a trend forecasting method with the Artificial Neural Network. In case of excess of the power generation by the renewable energy to demand it is converted to hydrogen with EL. In contrast, shortage of the electric power is made up with FC. The faster fluctuation power that cannot be compensated by the forecasting method is effectively compensated by SMES. In the ASPCS, the SMES coil with an $\mathrm{MgB}_{2}$ conductor is operated at $20 \mathrm{~K}$ by using liquid hydrogen supplied from a liquid hydrogen tank of the fuel cell vehicle station. The necessary storage capacity of SMES is estimated as $50 \mathrm{MJ}$ to $100 \mathrm{MJ}$ depending on the forecasting time for compensating fluctuation power of the rated wind power generation of 5.0 MW. As a safety case, a thermo-siphon cooling system is used to cool indirectly the $\mathrm{MgB}_{2}$ SMES coil by thermal conduction. In this paper, a trend forecasting result of output power of a wind power generation and the estimated storage capacity of SMES are reported.
\end{abstract}

Keywords: Renewable Energy; Artificial Neural Network; Forecasting; SMES; Liquid Hydrogen; Fuel Cell and Electrolyzer

\section{Introduction}

The applications of renewable energy are needed in the context of climate change and energy resource. But, when renewable energy generations, such as wind and photovoltaic power generations, affected by weather condition are introduced in large quantities, an existing power system may become unstable. Therefore, we propose a new system shown in Figure 1, called Advanced Superconducting Power Conditioning System (ASPCS) that is composed of Superconducting Magnetic Energy Storage (SMES), Fuel Cell-Electrolyzer (FC-EL), hydrogen storage and DC/DC and DC/AC converters in connection with a liquid hydrogen station for fuel cell vehicles [1-3].

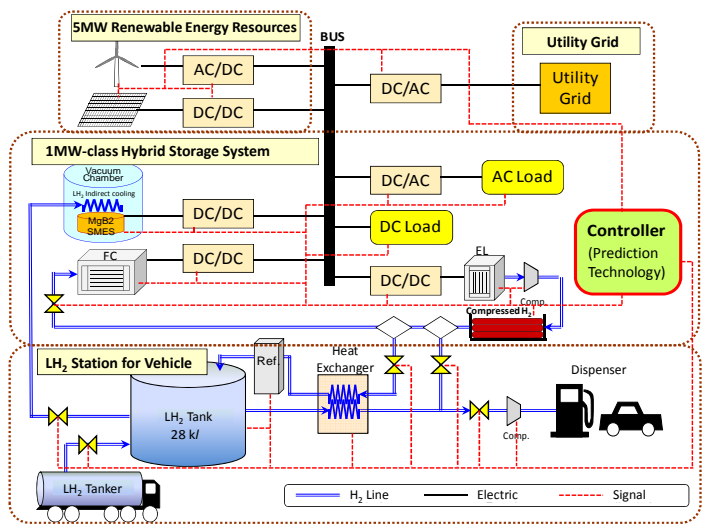

Figure 1. Advanced Superconducting Power Conditioning System. 
The ASPCS compensates the fast fluctuating electric power of the renewable energy sources by means of the SMES having characteristics of quick response and large Input-Output power, and hydrogen energy with FC-EL having characteristics of moderate response and large storage capacity. The moderate fluctuated power of the renewable energy is compensated by a trend forecasting with the Artificial Neural Network (ANN) [4, 5]. In case of excess of the power generation by the renewable energy to demand it is converted to hydrogen with EL. In contrast, in case of shortage the electric power is made up with FC. The faster fluctuation that cannot be compensated by the forecasting method is effectively compensated by SMES. In the ASPCS, the SMES coil with an $\mathrm{MgB}_{2}$ conductor is operated at $20 \mathrm{~K}$ by using liquid hydrogen supplied from a liquid hydrogen tank of the fuel cell vehicle station. The necessary storage capacity of SMES is estimated as $50 \mathrm{MJ}$ to $100 \mathrm{MJ}$ depending on the forecasting time for compensating fluctuation of the rated wind power generation of 5.0 MW. As a safety, a thermo-siphon cooling system is used to cool indirectly the $\mathrm{MgB}_{2}$ SMES coil by thermal conduction.

In this paper, a forecasting result of output power of a wind power generation and the necessary storage capacity of SMES are reported.

\section{Artificial Neural Network}

ANN is a model of biological neural network, and is composed of a lot of artificial neurons that collect output signals through a transfer function of Equation (1) when they are received input signals.

$$
f(x)=\tanh (x)=\frac{e^{x}-e^{-x}}{e^{x}+e^{-x}}
$$

The ANN shown in Figure 2 is a layered ANN. The layered ANN is formed three layers; an input layer that receives input signal, an output layer that produces outputs signals and a hidden layer that makes processes of signals.

Weights are set in connection between neurons. The outputs of each neuron inputs into other neurons after

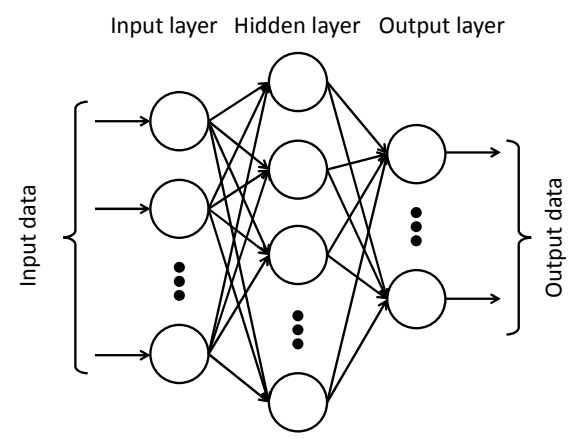

Figure 2. Layered ANN. they are multiplied by the weights. Since the weights are usually initialized with random values, they are adjusted to get desirable outputs. This is a learning process. A back propagation method that is one of the learning methods of ANN is used in this paper. The ANN can find relations between input data and the output data easily by the learning process.

\section{K-means Method}

A k-means method is one of clustering methods to perform data classification [6, 7]. And the method classifies given data into $\mathrm{k}$ clusters. The step of $\mathrm{k}$-means method is as follows:

(i) Determine number $\mathrm{k}$ of the clusters.

(ii) Assign clusters for the data at random.

(iii) Calculate the center of each cluster with the assigned data.

(iv) Calculate distances between the data and the cluster-centered, and assign the data to the nearest cluster again.

(v) When the allotment of all data into the clusters does not change by the process mentioned above, the calculation is over. Otherwise, the above process is repeated after the cluster centers are recalculated from newly assigned clusters.

\section{Simulation}

\subsection{Output Power Forecasting}

In this study, a wind power generation of rated output 5 MW having time series data of the output power shown in Figure $\mathbf{3}$ is used. The future trend output is forecasted for 50 seconds needed for control of FC-EL by the ANN model shown in Figure 4.

Let the output power time series of the wind power be $\left\{x_{1}, x_{2}, \ldots, x_{n}\right\}$. The ANN learns relation between $x_{t}$, $x_{t-10}, \ldots, x_{t-60}$ that are the output power at present time $t$ to 60 seconds before and $x_{t+50}$ that is the output power at 50

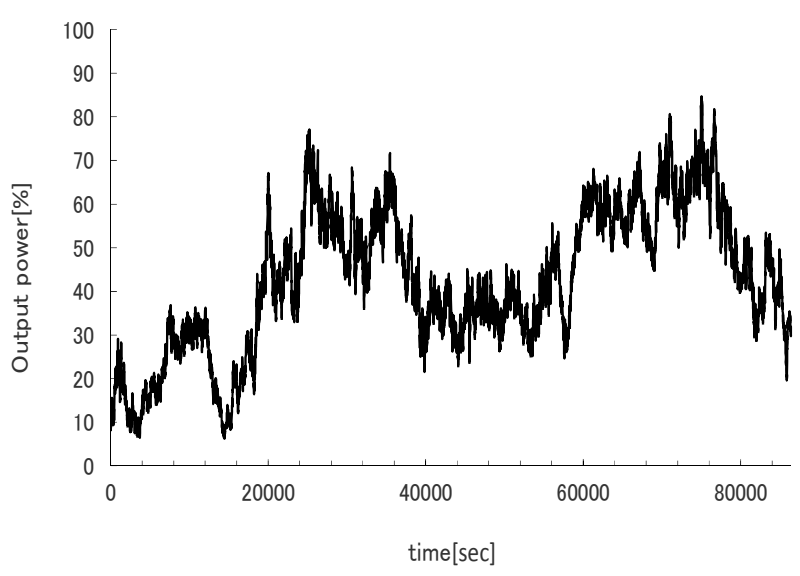

Figure 3. Output power of a wind power generation. 
seconds from the present time, and forecasts output power 50 seconds in future. The forecasting result by the ANN is shown in Figures 5, 6 and Table 1. Table 1 includes the result of moving average that is calculated by $x_{t}=\left(x_{t-10}+x_{t-20}+x_{t-30}+x_{t-40}+x_{t-50}\right) / 6$ to compare with the result by the ANN. The ANN can forecast the output power of the wind power generation at 50 seconds in future, and it is shown that the result of the ANN is better than that of the moving average.

\subsection{The Necessary Storage Capacity of SMES}

When FC-EL is operated based on the forecast mentioned above, charging or discharging power by SMES compensates the difference between actual output power and the forecasted output power as shown in Figure 7. The required storage capacity of SMES shown in Table $\mathbf{2}$ is determined by alternately charging or discharging power. In addition, distribution of the charging or discharging power by SMES is shown in Figures 8 and 9. From the above result, the storage capacity of SMES using the ANN is smaller than that using moving average.

\section{Conclusions}

In this paper, the output power of the wind power generation is forecasted for ASPCS that is composed wind

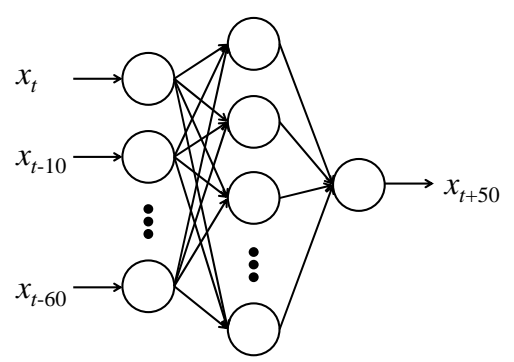

Figure 4. The ANN forecasting output power of a wind power generation.

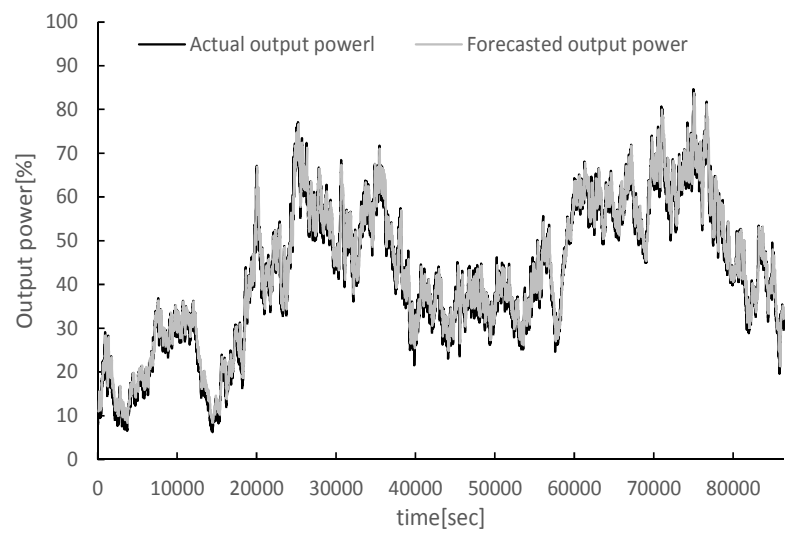

Figure 5. Forecasting result of output power of a wind power generation by ANN.

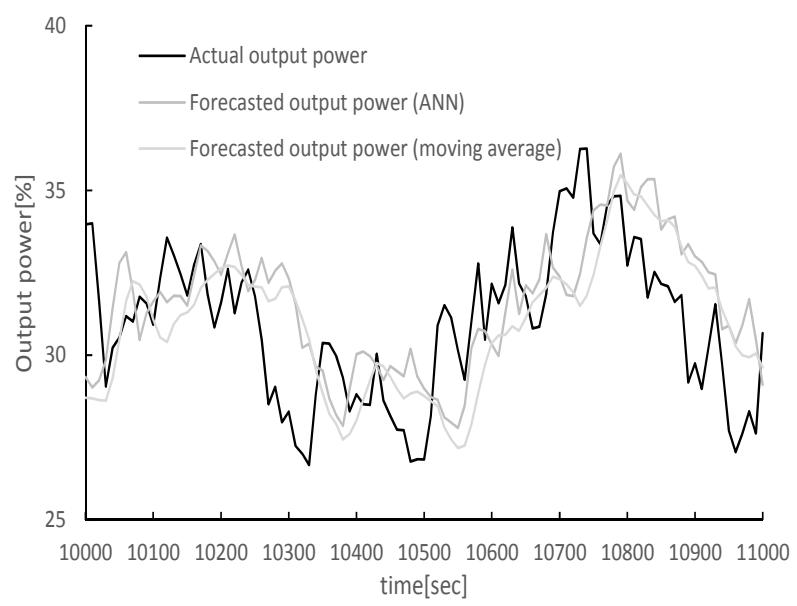

Figure 6. Forecasting result of output power of a wind power generation by ANN (between 10000 and 11000 seconds).

Table 1. Forecasting result of output power of a wind power generation.

\begin{tabular}{ccc}
\hline Forecasting method & ANN & Moving average \\
\hline Average error [MW] & 0.028 & -0.001 \\
Distribution [(MW) $\left.{ }^{2}\right]$ & 0.025 & 0.028 \\
Maximum absolute error [MW] & 0.685 & 0.725 \\
\hline
\end{tabular}

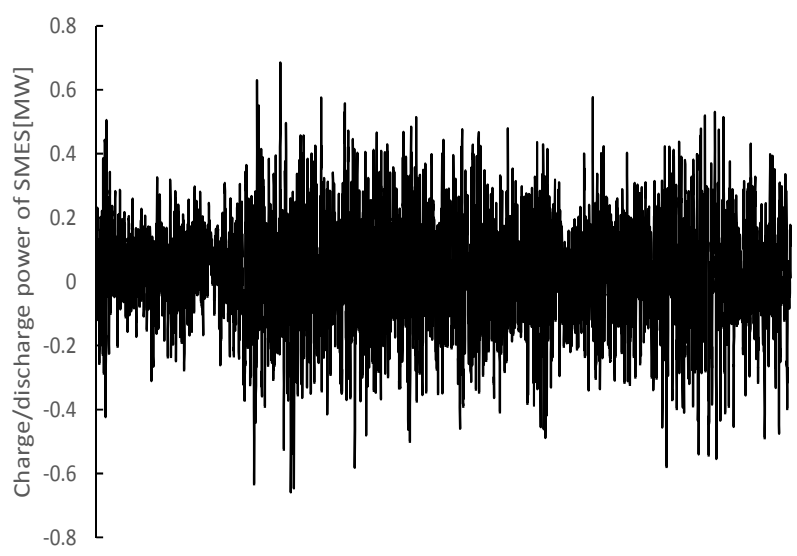

Figure 7. Charge/discharge power of SMES.

Table 2. Storage capacity of SMES.

\begin{tabular}{ccc}
\hline Forecasting method & ANN & Moving average \\
\hline Maximum charge capacity[MJ] & 72.0 & 73.5 \\
Maximum discharge capacity[MJ] & -61.8 & -83.4 \\
Average[MJ] & 1.45 & -0.05 \\
Absolute average[MJ] & 6.47 & 7.83 \\
\hline
\end{tabular}




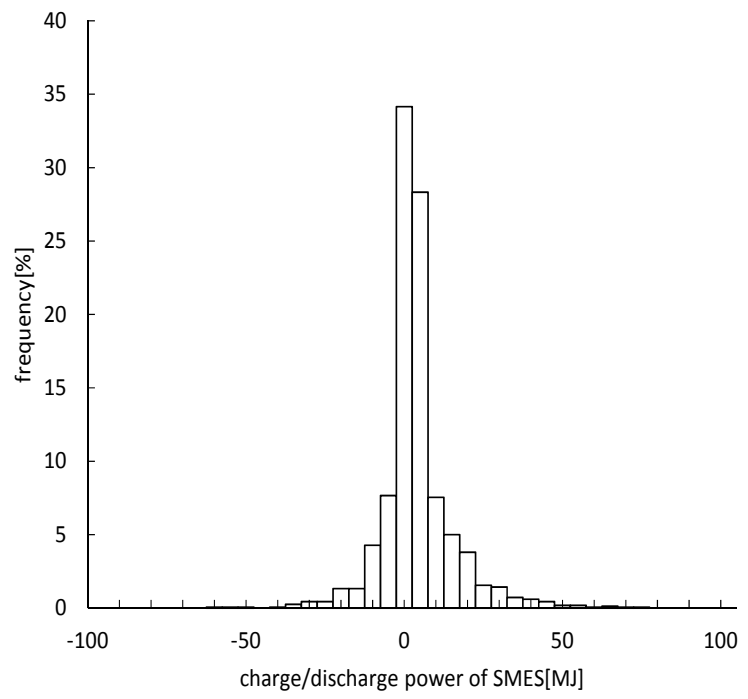

Figure 8. Distribution of charging/discharging energy of SMES (ANN).

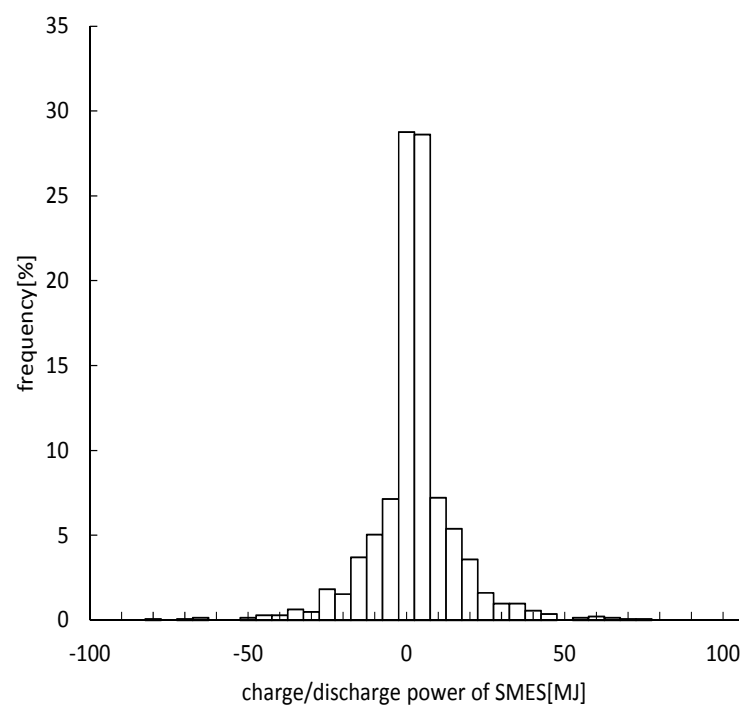

Figure 9. Distribution of charging/discharging energy of SMES (moving average). power generation, SMES and FC-EL and supplies smoothed power from this system. As a result, the ANN has better accuracy than the moving average method and is able to forecast the output power, and the required storage capacity of SMES is smaller. In the future study, we optimize structure of the ANN and improve the selection method of learning data.

\section{Acknowledgements}

This work is supported by the Advanced Low Carbon Reduction Technology R\&D of Japan Science and Technology Agency.

\section{REFERENCES}

[1] T. Hamajima, et al., "Application of SMES and Fuel Cell System Combined with Liquid Hydrogen Vehicle Station to Renewable Energy Control,” IEEE Transactions on Applied Superconductivity, Vol. 22, No. 3, 2011, p. 5701704. doi:10.1109/TASC.2011.2175687

[2] T. Shintomi, et al., "Design Study of SMES System Cooled by Thermo-siphon with Liquid Hydrogen for Effective Use of Renewable Energy,” IEEE Transaction Supercond., Vol. 22, No. 3, 2011, p. 5701604. doi:10.1109/TASC.2011.2178575

[3] T. Nakayama, et al., "Optimization of SMES Compensation Capacity for Stochastic Power Using a Kalman Filter,” TEION KOGAKU, Vol. 45, No. 3, 2010, pp. 99-106. doi:10.2221/jcsj.45.99

[4] D. E. Rumelhart, et al., "Parallel Distributed Processing," MIT Press, 1986.

[5] T. J. Sejnowski, et al., "Parallel Networks that Learn to Pronounce English Text,” Complex System, Vol. 1,1987, pp. 145-168.

[6] J. B. MacQueen, "Some Methods of Classification and Analysis of Multivariate Observations," Proceedings of $5^{\text {th }}$ Berkeley Symposium on Math, Stat. and Prob., 1967, pp. 281-297.

[7] E. W. Forgy, "Cluster Analysis of Multivariate data: efficiency vs. Interpretability of Classifications,” Biometrics, Vol. 21, 1965, pp. 768-769. 